Noguera Muntadas, Montserrat.

Profesora titular de Medios Informáticos en ESDAP (Escuela Superior de Diseño y Artes Plásticas de Cataluña). Campus Llotja.

Cursando el Máster de Producción e Investigación artística en la Universidad de Barcelona. Facultad de Bellas Artes.

\title{
Imaginando procesos para el aprendizaje vectorial
}

\section{Imagining processes for vector learning}

TIPO DE TRABAJO: Comunicación.

PALABRAS CLAVE

Vectorial, Vector, Trazo, Dibujo, Educación, Proceso.

KEY WORDS

Vector, Stroke, Education, Draw, Process.

RESUMEN

Esta propuesta se centra en el enfoque educativo del espacio vectorial a partir de la investigación gráfica y formal de mi obra. En este sentido es una investigación para el arte. Desde mediados de los años 80 , el entorno vectorial empezó a introducirse en la educación artística y ha permanecido inmerso en un ensayo educativo, en un tanteo formal, buscando un lugar dentro de los modos actuales de producción. Con la perspectiva que nos ofrece el tiempo y las redes sociales, observamos el imaginario colectivo. Expongo una reflexión sobre el giro teórico y de proceso que supone una mirada experimental que busca un más allá de este imaginario a través de métodos para el desarrollo de una verdadera inteligencia plástica de ese entorno.

La investigación práctica se inició con el estudio del trazo como elemento humano de expresión y comunicación. A partir de una visión histórica, considero ciertas herramientas vectoriales como una cola de cometa de nuestro legado. Es importante hablar de lo que hay de eterno en el gesto humano de trazar trasladado en el sí de esas tecnologías y cómo convive con otros procesos cognitivos nuevos y propios de la naturaleza digital, los cuales precisan de una instrucción específica.

Las metodologías de aprendizaje son el eje vertebrador para una educación que busca sensibilizar la tecnología y observar en ella la continuación del deseo humano de trazar. En la investigación, el juego es entendido no solo como método de experimentación previo al dibujo y a la creación, sino como proceso intrínseco del trabajo y la cultura gráfica. Las artes combinatorias, la repetición, la exploración de la grafomotricidad a modo de tratado son pilares de los procesos investigados. La educación desde esta perspectiva ofrece un modelo de sensibilización de la técnica, una estructura de conocimiento y una preparación para el desarrollo de las posibilidades expresivas. Una experimentación y conocimiento profundo permite el uso del espacio vectorial en un contexto más amplio de investigación artística actuando como soporte formal a un marco conceptual.

\section{ABSTRACT}

This proposal focuses on the educational approach of the vector space from the graphic and formal research of my work. In this sense it is an investigation for art. Since the mid 80 's, the vector environment began to be introduced into art education and has remained immersed in an educational experimentation, in a formal trial, looking for a place within the current ways of production. With the perspective offered by time and social networks, we observe the collective imaginary. I reflect on the theoretical and procedural turn derived from an experimental vision that looks beyond this imaginary through methods that lead to the development of a true plastic intelligence in that environment.

Practical research started with the study of stroke as a human element of expression and communication. From a historical perspective, I consider certain vector tools as comet tails of our legacy. It is important to talk about what is eternal in the human 
gesture of drawing transferred to the core of these technologies and how it coexists with other new and specific cognitive processes of the digital nature, which require specific instruction.

Learning methodologies are the backbone of an education that seeks to sensitize technology and observe in it the continuity of the human desire to trace. In research, playing is understood not only as a method of experimentation prior to drawing and creation, but as an intrinsic process of work and graphic culture. Combined arts, repetition and the exploration of graphomotor skills as a discourse are the pillars of the investigated processes. Education from this perspective offers a model of awareness of the technique, a structure of knowledge and a preparation for the development of expressive possibilities. Experimentation and deep knowledge allow the use of vector space in a broader context of artistic research acting as formal support to a conceptual framework.

\section{INTRODUCCIÓN}

A mediados de los años 80 surgieron nuevos medios para la producción gráfica y se sumergieron en variadas disciplinas, especialmente en el diseño y la ilustración. La editorial Inforbook's me ofreció la oportunidad de escribir sobre el entorno vectorial con fines a un conocimiento fundamentalmente técnico, enfoque necesario por la reciente llegada del programario. En el mundo del arte, algunos de estos entornos gráficos permanecieron más tiempo en el campo de lo foráneo e incluso como ajenos a lo artístico. El tiempo transcurrido hasta hoy y la extensiva práctica de esa técnica permiten una perspectiva del imaginario colectivo, los usos, los mundos visuales y las cualidades formales estandarizadas.

Mi investigación gráfica en el espacio vectorial se inició con la práctica de estilos y posibilidades gráficas afines a un primer encuentro con el vector, pero más adelante me interesé por la búsqueda de cualidades expresivas que superaran ese primer estadio y el mundo visual supuesto, deseaba situarme en un plano creativo donde la herramienta empezara a desaparecer. Con ello se propone una reflexión sobre el giro teórico y de proceso que supone una mirada experimental más allá del imaginario colectivo instalado, y la puesta en valor de una educación que estime de donde venimos y a donde vamos, profundizando en lo intrínseco de la naturaleza digital vectorial. Uso el término "espacio o entorno vectorial" para referirme a todos los programas vectoriales, sus interfaces y el conjunto de funciones.

Muchos autores han descrito las ventajas relacionadas con la información digital en términos de facilidad. Codina (2000: 71), desde sus estudios sobre el libro digital, habla de algunas cualidades de lo digital y apunta sobre la reusabilidad como "la propiedad que presenta la información digital de poder ser fácilmente seleccionable, recuperable, reproducible y transportable". Hemingway (citado por Codina, 2000: 70) expone, con respecto a la creación, que "con medios digitales, el usuario puede manipular o cambiar elementos individuales y crear nuevos elementos mucho más fácilmente que con medios analógicos". Esa facilidad tan real y valorada por tantos otros es el sustrato que permite la verdadera experimentación formal, pero, en realidad, no es más que eso. En un primer encuentro con la tecnología vectorial, esa facilidad puede incluso operar, juntamente con las posibilidades múltiples del programa, a modo de efecto potente que domina toda intención expresiva. La práctica desvela que el acercamiento al espacio vectorial y a su aprendizaje profundo, así como el uso singular y personalizado de su expresividad, no es especialmente ni fácil ni rápido, pero su encuentro abre horizontes inesperados a modo de recompensa.

\section{METODOLOGÍA}

Los inicios de la investigación se centraron en la observación, deducción y traslación de aspectos formales de nuestro legado artístico a la naturaleza y construcción del espacio vectorial, un ensayo de procesos y resultados. El estudio del trazo cobró una especial relevancia por ser entendido como gesto humano elemental de comunicación y por las variables expresivas de la línea que el espacio vectorial incorpora. En un momento más avanzado, en el deseo de sistematizar procesos y compartir ese conocimiento, me centré en la búsqueda de metodologías para el aprendizaje y la demostración, trabajando a modo de metametodología.

La relación entre el conocimiento gráfico tradicional y la reflexión sobre cómo innovar fue un eje importante.

Nuestras instituciones y nuestros saberes son métodos y formas para ayudarnos a caminar en la ignorancia. Pero cuando convertimos sus aserciones en verdades, la ignorancia deviene estupidez. Los bastones se vuelven contra nosotros formando empalizadas y jaulas o convirtiéndose en armas destructivas, y los muros empiezan a oprimirnos. (Maillard, 2009: 10).

La clásica gramática visual podía trabajar conjuntamente e interactuar con las funciones básicas del entorno vectorial, convirtiendo el espacio virtual en un lugar de desarrollo de la inteligencia gráfica con una fluidez continua entre la máquina y el pensamiento, este fue el paso previo a la investigación formal y el sustrato que la permitía. Por otra parte, el conocimiento tradicional era una fuente de recursos formales y estéticos. Estas eran las fuentes de nuestros saberes, pero en todos los procesos he evitado siempre el muro que 
nos oprime: el esbozo predeterminado y el calco sistemático. He optado, a cambio, por alcanzar aquello no previsto o el crecimiento de lo previamente preconcebido, así como la introducción y gestión del azar, el estudio de la repetición y otros procesos alternativos. En conjunto, es imprescindible el errabundeo gráfico, el errar continuado que busca lugares del no ordenamiento para encontrar puntos de desarrollo de una inteligencia acorde a las posibilidades de ejecución del espacio vectorial. Dos son los pasos imprescindibles antes de la creación: comprensión de la construcción vector y errar en la experimentación.

Las metodologías de aprendizaje enmarcadas en esta investigación formal emprendieron, cada vez más, una visión lúdica, entendida no solo como fase de entrenamiento y acercamiento, sino como una cualidad inextricable del propio espacio vectorial y de la cultura gráfica. El juego, en la metodología de aprendizaje y de trabajo, constituye un fundamento, y cuanto más avanzamos en la comprensión del espacio vectorial, más se pone en evidencia su profunda dependencia. La pantalla ha sido el campo de juego que, al ser virtual, ayuda a ejercer una función en principio desvinculada de lo "real".

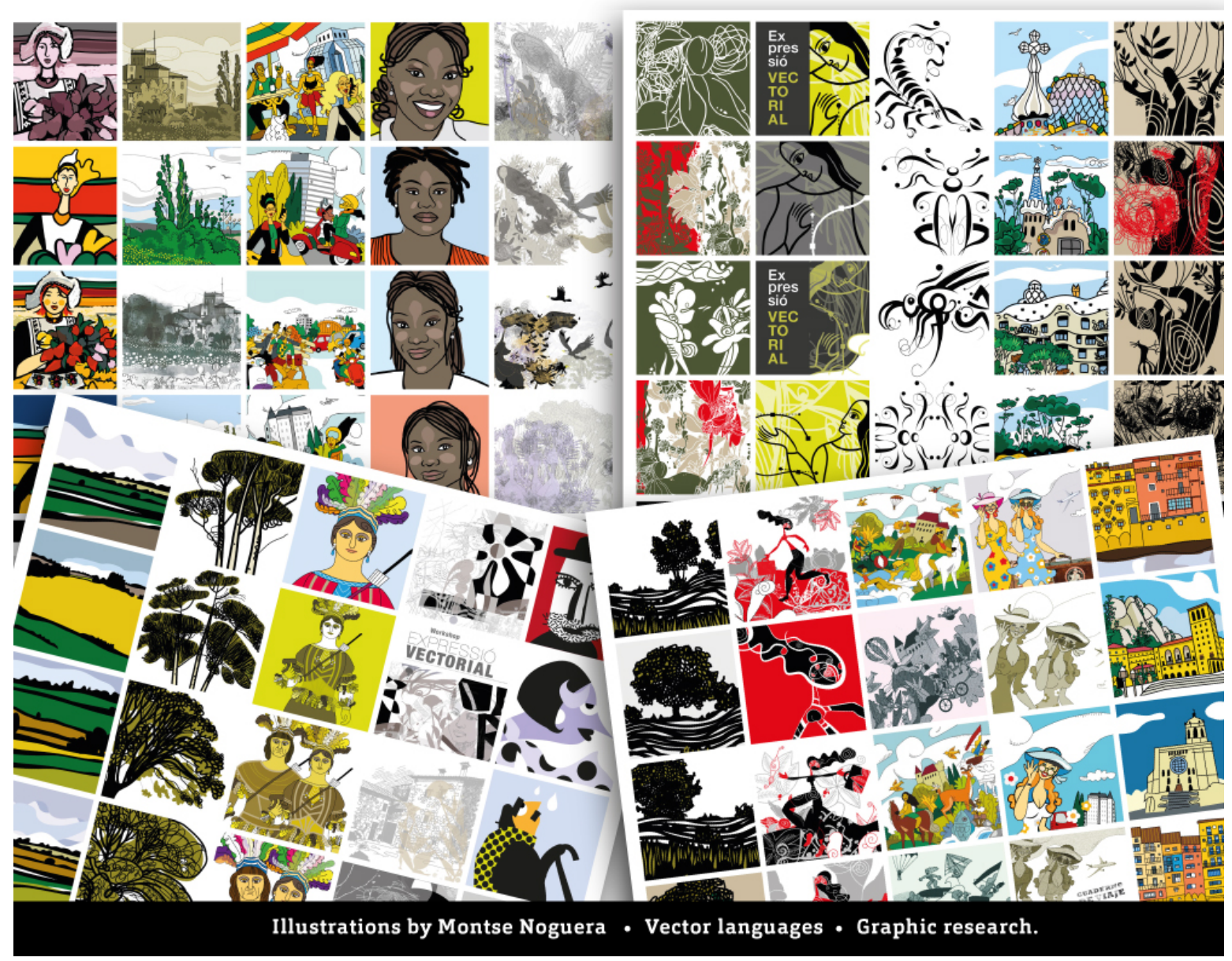

Figura 1. Mundos visuales del espacio vectorial. Contornos definidos, áreas planas de color y otras características definen el imaginario y el primer estadio de encuentro con el espacio vectorial. Montse Noguera, 1997-2015.

\section{DESARROLLO}

1. El gesto humano de trazar. Un avance cognitivo en el entorno vectorial.

El hombre ha trazado con todos los medios a su alcance. El trazo es un elemento básico de comunicación humana:

El trazo es signo del gesto, y el gesto es propio del hombre frente a la máquina. El trazo remite a lo humano frente a lo mecánico. [...]. El trazo, en resumen, es el artificio gráfico que condensa los valores semánticos que componen el estereotipo social más extendido sobre lo artístico: lo humano, lo gestual, lo original, lo creativo, lo imprevisible, lo impulsivo, lo único, lo irrepetible, lo plástico, lo lúdico, lo gratuito... (Ruíz Collantes, 1993: 121, 122). 
Remitir a lo humano buscando una plasticidad es un motor para la práctica. Las vinculaciones con nuestro conocimiento histórico en técnicas gráficas aportan fuentes de referencia.

El vector revela ciertos vínculos plásticos e intelectuales con el grabado. Pla (1956) expone el esfuerzo que representa adquirir el oficio y dominar los secretos de la acción de los ácidos, los barnices y las puntas de acero en el grabado calcográfico. El vector no es tampoco una técnica directa, apuntamos con ello a todos los conocimientos e instrucciones que el creador debe adquirir y practicar previamente a la obra, y al carácter del trabajo. Hay una vinculación interesante con el grabado observando una similitud formal en las maneras de tratar el blanco y el negro. Podemos conseguir efectos a base de tramado que pueden compararse con las mordidas y la formas de conseguir grises y sombreados. Los grabados son enriquecedores en la observación de los modos de trazar, aportan recursos para descubrir posibilidades expresivas extrapolables al vector y así crecer plásticamente. Las xilografías son también un marco de sugerencias plásticas. Pero el vector es capaz de desprenderse en su momento de esas observaciones, arrancando de nuestro pasado para revelarse en otra dimensión donde aporta un horizonte plástico propio y nuevo a explorar.

La caligrafía china sostiene la variedad en la unidad y es rica en el estudio del trazo:

El trazo será la piedra basal sobre la cual China construirá su Pintura. Artistas de diferentes épocas y temperamentos darán cuenta en sus obras de las múltiples posibilidades expresivas del Trazo asociado al Vacío, bien desde sus vertientes más contenidas hasta sus manifestaciones más excéntricas y extrovertidas. De conformidad con ello surgirían diferentes modalidades del trazo, destinadas a cubrir distintas necesidades expresivas. (Tegaldo, 2013: 21).

Las variantes expresivas de la línea vectorial son afines al trazo de la caligrafía china que se explica en términos de trazo de contorno, trazo homogéneo con carácter contenido y controlado, trazo modulado con carácter rítmico y dinámico, trazo con volumen y trazo único o de carácter sintético, todas ellas vinculadas a efectos de expresión. La caligrafía china tiene nombres para todas las variedades y variables técnicas en el manejo del pincel, seria largo y extenso, pero pone de manifiesto su amplia cultura en el trazo.

\subsection{La diferencia del trazar en el espacio en vectorial.}

Ruiz Collantes describe algunas dimensiones del trazo:

El trazo supone un cierto grado de libertad en el instante de la realización y en el resultado final. En la producción del trazo, si el sujeto no la ha automatizado por la repetición, se pone en juego la valentía del autor. No hay un control absoluto sobre el proceso y la conclusión. Es como un saltar al vacío a partir de una decisión y de un impulso. Un salto que puede salir bien o mal. Es posible determinar el punto de partida, el punto de llegada y la dirección que hay que seguir para llegar de uno a otro. Pero el recorrido se ejecuta en caída libre. (1993: 121).

Los valores de gesto, trazo, libertad y incertidumbre se remodelan adquiriendo prácticas y procesos diferentes en el espacio vectorial. En los medios tradicionales, el trazo es signo del gesto, y el gesto es más corporal que mental. El brazo y la mano se mueven creando el gesto, predominando el cuerpo sobre la mente. En el espacio vectorial, el acto de creación del trazo puede ser corporal, pero es especialmente interesante y evolutivo entender que los procesos vectoriales son principalmente mentales, reflexivos y cognitivos. Un gesto puede ser resultado de procesos predominantemente mentales y no perder su carácter de gesto. Esta es una de las diferencias que implica un especial giro teórico, procesal y conceptual. No por ello olvidamos el gesto manual de trazar en tableta o en pantalla, pero éste será solo un primer paso que nos ha de permitir entrar en las puertas de su uso pleno. Ese aspecto no desvaloriza el trazo, al contrario, se adquiere la posibilidad de la espontaneidad surgida del gesto o surgida de otras dimensiones digitales que conectan y operan en nuestro pensamiento.

Un trazo vectorial es una línea matemática suspendida entre puntos, puede variar, remodelarse en cualquier momento, es un elemento vivo en toda su constante potencia. Las operaciones básicas de gramática visual: giro, ampliaciones, reducciones, repeticiones, superposiciones, etc., lo convierten en un elemento plástico dentro del espacio virtual. El trazo vector puede ser revivido y remodelado en todo momento. 

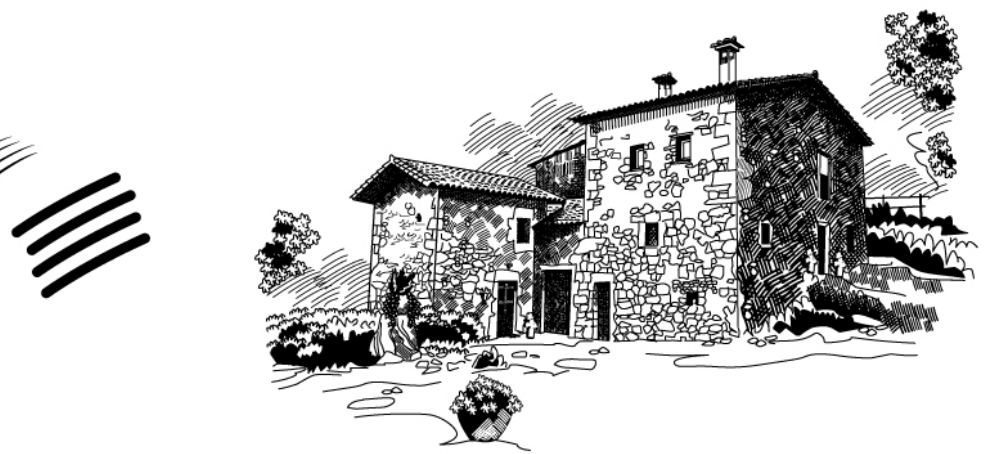

Figura 2. Dibujo en pantalla con pluma vectorial y trazos, gestión de grupos y manipulaciones básicas. "Cal Tracanet”. Montse Noguera, 1999. Elaboración propia.
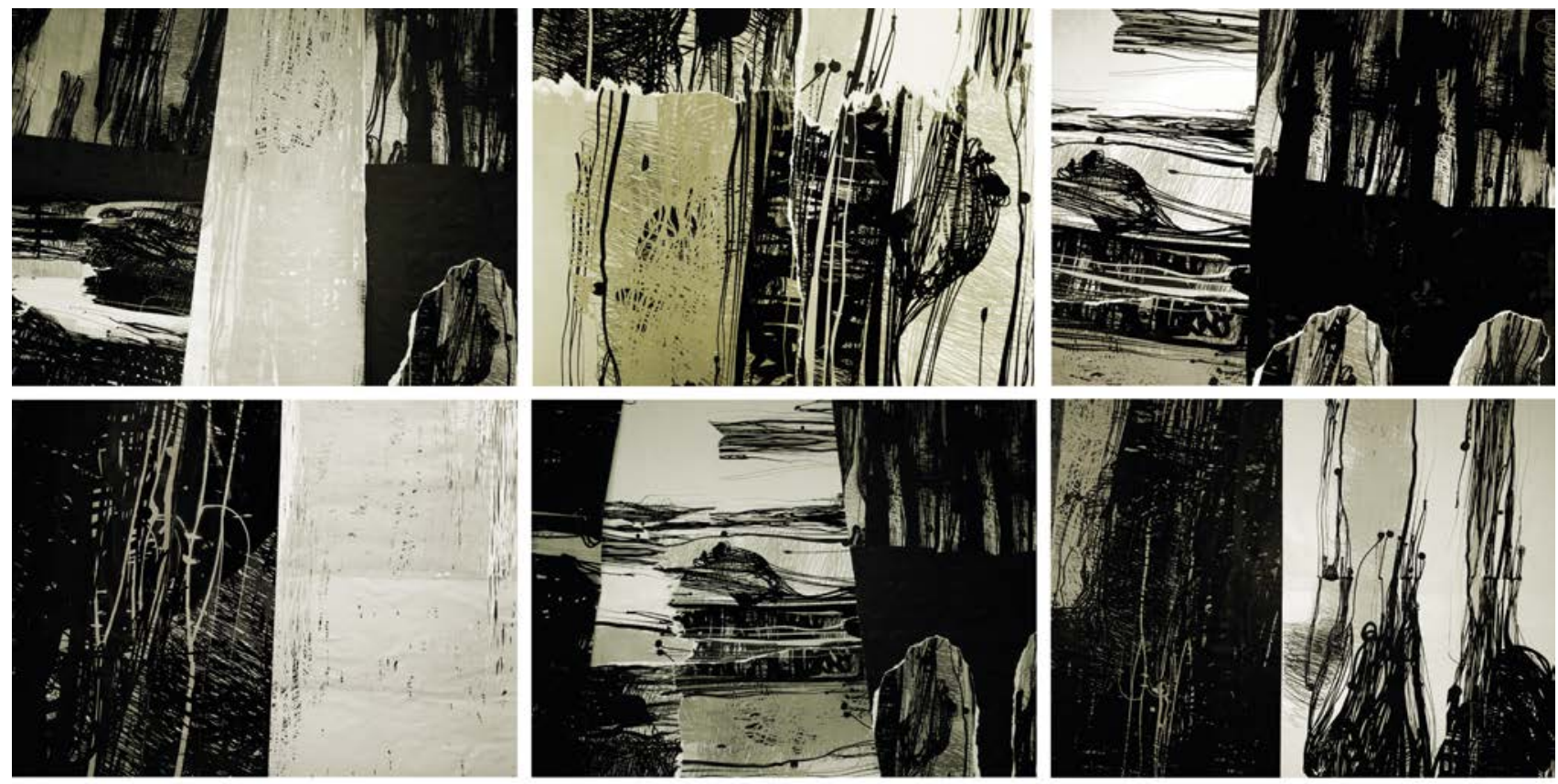

\section{BOSQUES NEGROS. Obra de Montse Noguera Muntadas.}

Figura 3. Una obra artística en un conjunto de búsquedas que fluye de inquietudes subjetivas y se sumerge en una exploración formal. El proceso gráfico y los resultados amplían y extrañan nuestro imaginario sobre las posibilidades del entorno vector y de como nos acercamos a él. "Bosques negros". Montse Noguera, 2018. Elaboración propia. 

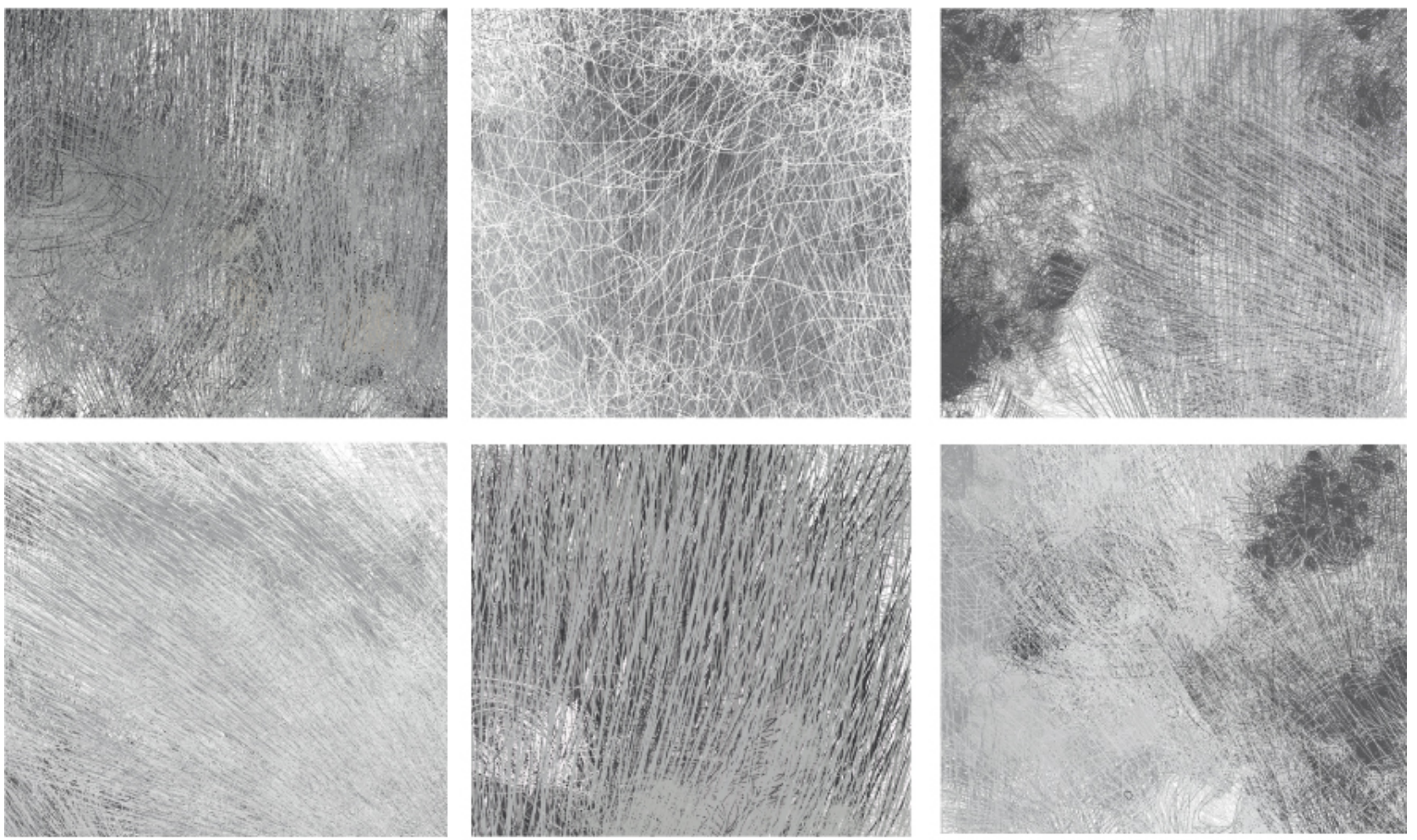

Detalles de Fields and forests. Obra de Montse Noguera Muntadas.

Figura 4. Un juego de grises, repeticiones y superposiciones crea el efecto de trazado. El trabajo acciona un proceso repetitivo con gestión de grupos y tonos, estructurado a partir de unos trazos iniciales. Detalles. "Fields and forests". Montse Noguera, 2017-2018. Elaboración propia. 

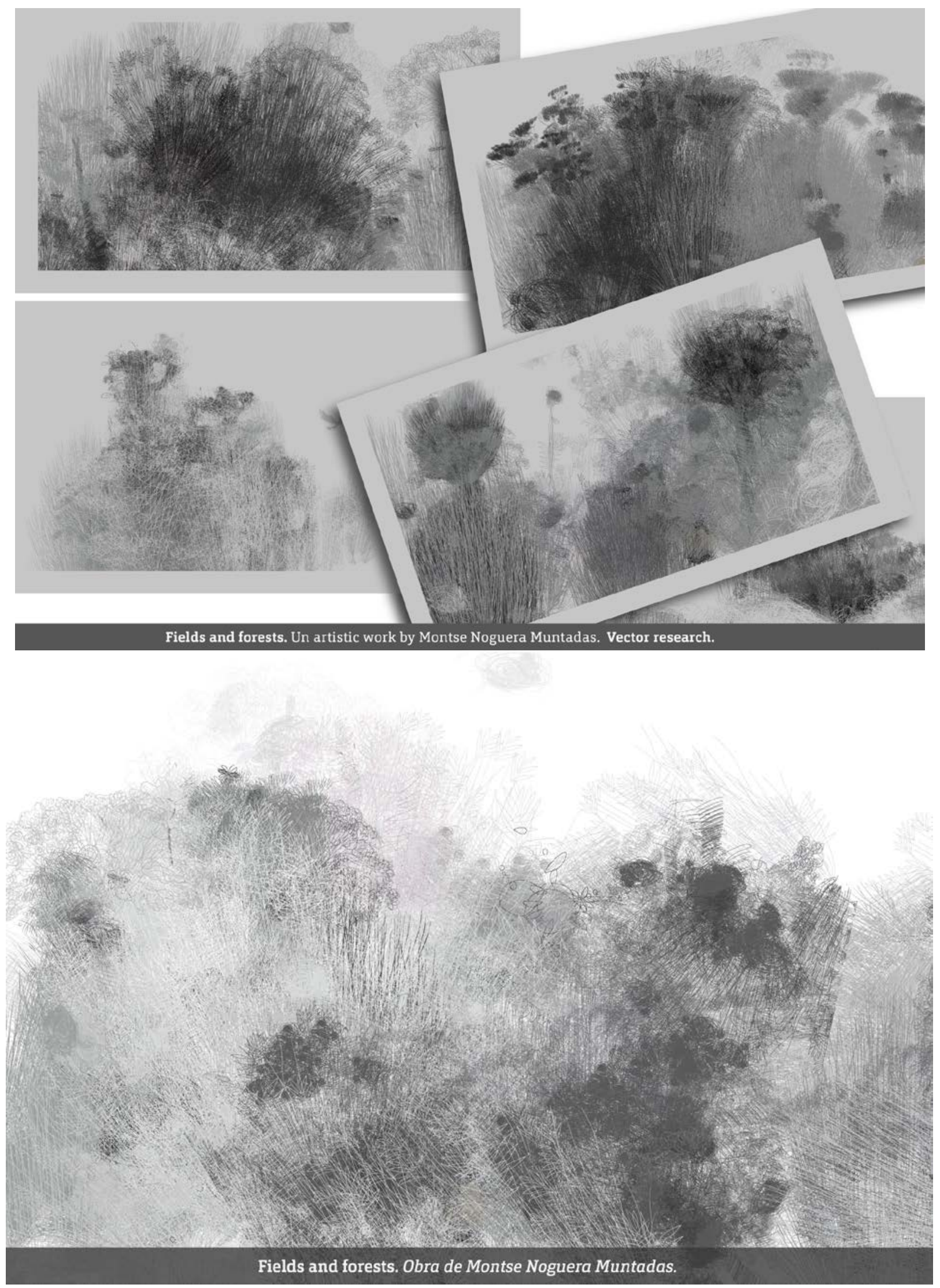

Figura 5 y 6. Representaciones difusas del tercer paisaje. "Fields and forests". Montse Noguera, 2018. Elaboración propia. 


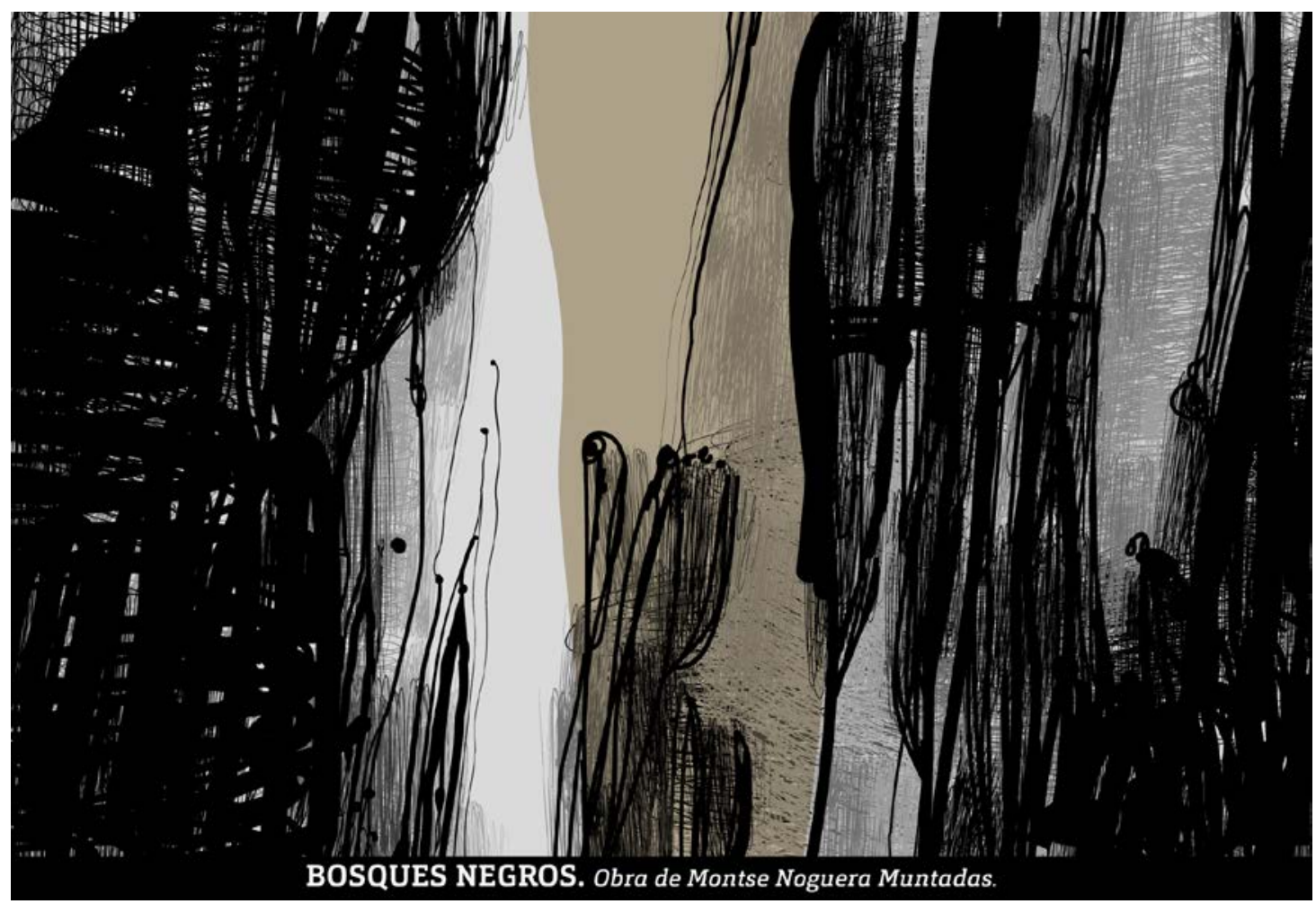

Figura 7. Trabajo partir de trazos gruesos, expansiones, recortes, repeticiones, cambios de color y superposiciones, buscando efectos pictóricos. "Bosques negros". Montse Noguera, 2018. Elaboración propia.
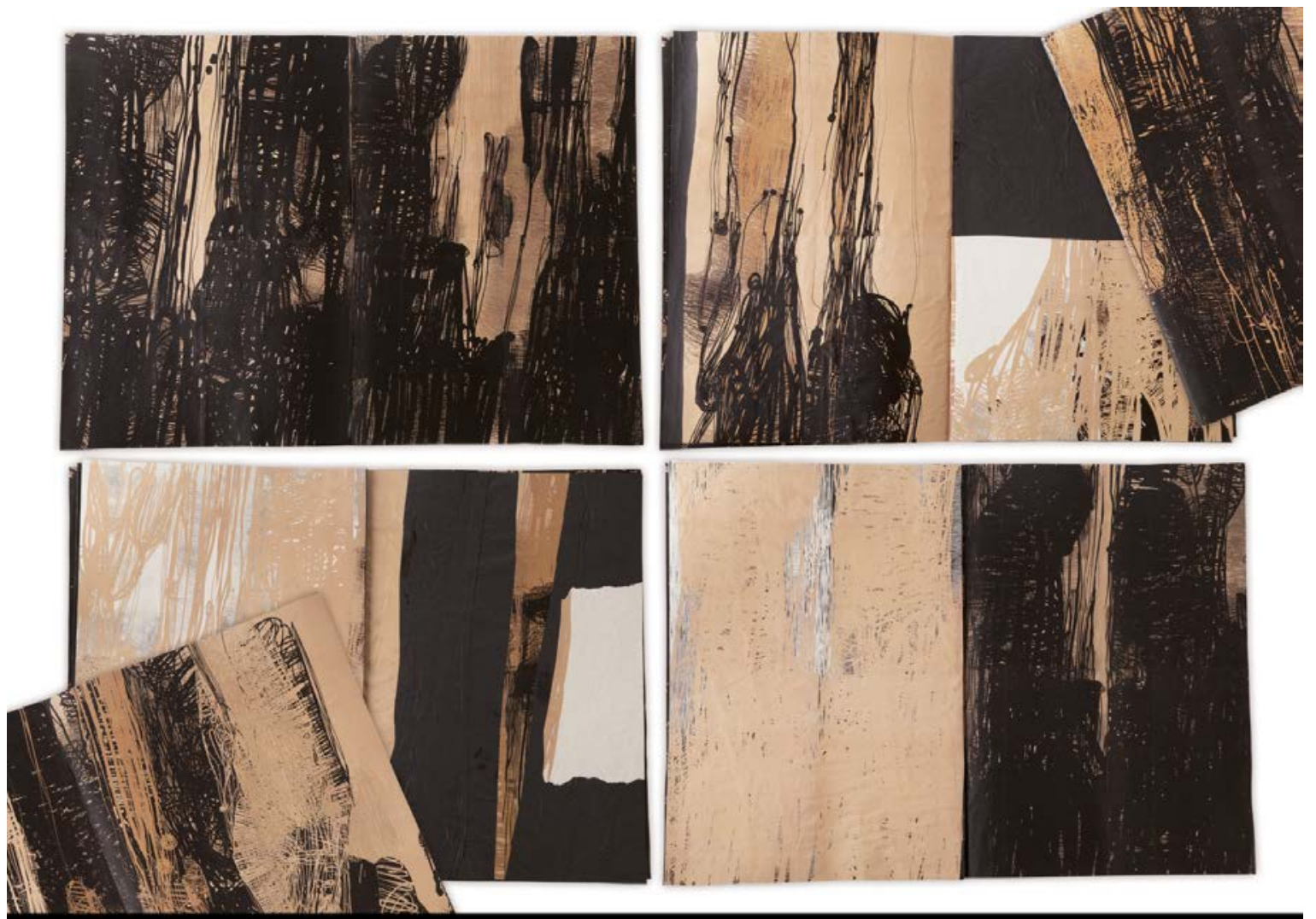

Book of forests and the passage of time - Artist book by Montse Noguera - Barcelona 2018

Figura 8. Libro de artista. Obra presentada en el Máster de producción e investigación artística en la Universidad de Barcelona. El espacio vectorial funciona como soporte al marco conceptual. "Book of forest and the passage of time". Montse Noguera, 2018. Elaboración propia. 


\section{Vector ludens. El juego en los procesos gráficos}

El grado de implicación del juego en nuestras actividades culturales queda patente en los estudios de Huizinga y en la concreción: "Porque no se trata, para mí, del lugar que al juego corresponda entre las demás manifestaciones de la cultura, sino en qué grado la cultura misma ofrece un carácter de juego" (Huizinga, trad. de Imaz, 2007: 9). Se podría establecer una afirmación paralela en relación con el estudio que nos ocupa. El artista que juega con sus herramientas ejerce una función tan elemental como la de crear en si, y ya no estamos tratando del lugar que ocupa el juego en la experimentación formal vectorial, sino en qué grado el mismo entorno vectorial ofrece, en la comprensión más profunda de su naturaleza, un carácter de juego concebido como fenómeno gráfico y de proceso. El juego gráfico entendido como cultura, el juego como cualidad determinante de la creación. Las grandes columnas en que se cimienta el entorno vectorial están impregnadas de juego, de reglas internas donde brota un espíritu inventivo, entre la seriedad y la broma en términos de comprensión del juego de Huizinga. Podemos pensar que, al igual que grandes ámbitos de la humanidad, el vector y su filosofía de trabajo hunde sus raíces en el terreno de la actividad lúdica.

El concepto juego en el entorno vectorial conecta de manera especial. "Dentro del campo de juego existe un orden propio y absoluto. He aquí otro rasgo del juego: crea orden, es orden" (Huizinga, 2007: 24). En el entorno vectorial el orden en los procesos adquiere una relevancia especial, la exploración en procesos no está exenta de órdenes y reglas. Otras situaciones que se dan al juego son tensión, incertidumbre y azar, estrechos vínculos necesarios para cumplir con el objetivo de huir del esbozo o calcos predeterminantes.

Veamos donde radica, según Huizinga, la libertad de la actividad lúdica:

Todo juego es, antes que nada, una actividad libre. El juego por mandato no es juego, todo lo más una replica, por encargo, de juego [...]. El niño y el animal juegan porque encuentran gusto en ello, y en esto consiste precisamente su libertad [...]. Se juega en tiempo de ocio. Sólo secundariamente, al convertirse en función cultural veremos los conceptos de deber y tarea vinculados al juego. (2007: 20).

Se podría pensar, en un principio, que el juego incluido en las metodologías de aprendizaje seria algo como un encargo de juego. Pero esta no seria su lectura, sino que en el juego del aprendizaje se encuentra precisamente el sentido mismo de juego inextricable a las tareas creativas. Pero esa libertad inicial tiene su sentido, los principios de mi investigación se desarrollaron fuera de las tareas habituales, de las aulas y del encargo, en un contexto de total libertad; fue esa libertad la que habría de dar sus frutos y vislumbrar la vinculación entre trabajo gráfico y juego, y después entre juego y aprendizaje.

El carácter lúdico es sublime, nada desmerece porque adquiera carácter de juego, sino que "éste queda exaltado porque su concepto se eleva hasta las regiones más altas del espíritu". (Huizinga, 2007: 35). El juego se sigue practicando con seriedad y entusiasmo aún con la conciencia de saber que se está jugando.

El proyecto "A few traces, a thousand faces" de las figuras 9-12 es un trabajo artístico que juega con la combinatoria a la vez que busca un uso especial del trazo. Se conduce así a una interesante y divertida demostración sobre la capacidad de expresión gráfica del espacio vectorial. El proyecto empezó con el deseo de buscar un toque de acabado orgánico en el trazo vectorial. En una primera intención, se crearon unos trazos y empezó el juego de combinatoria con métodos heurísticos de ensayo error a través de funciones elementales de gramática visual, tales como: ampliaciones, reducciones, giros, reflejos, superposiciones, cortes, etc. En la gestión digital de los trazos, las propiedades del ser digital también estuvieron presentes en términos de reutilización, recuperabilidad, computabilidad y virtualidad, trabajando en pantalla. El resultado de este primer nivel de actuación fue un grupo de retratos. Este primer grupo actuó como base para continuar jugando a través de interacciones entre ellos, algunas reglas y órdenes internas condujeron a la multiplicación de resultados hacia un segundo nivel de creación. Finalmente, la visualización de contraformas entre trazos generó un tercer nivel de resultados.

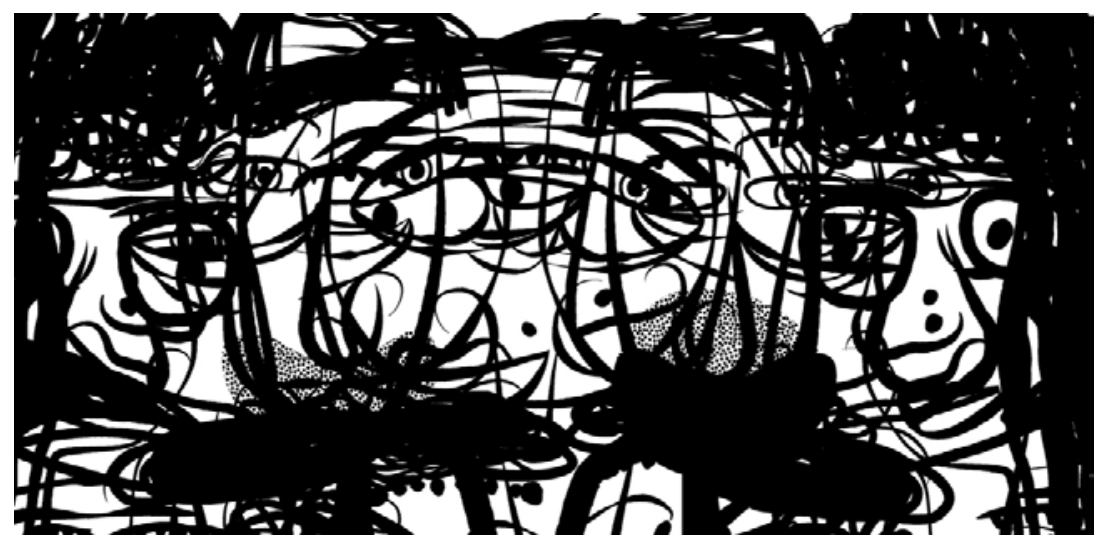

Figuras 9. Detalle de mezcla de trazos. "A few traces, a thousand faces". Montse Noguera, 2015. Elaboración propia. 


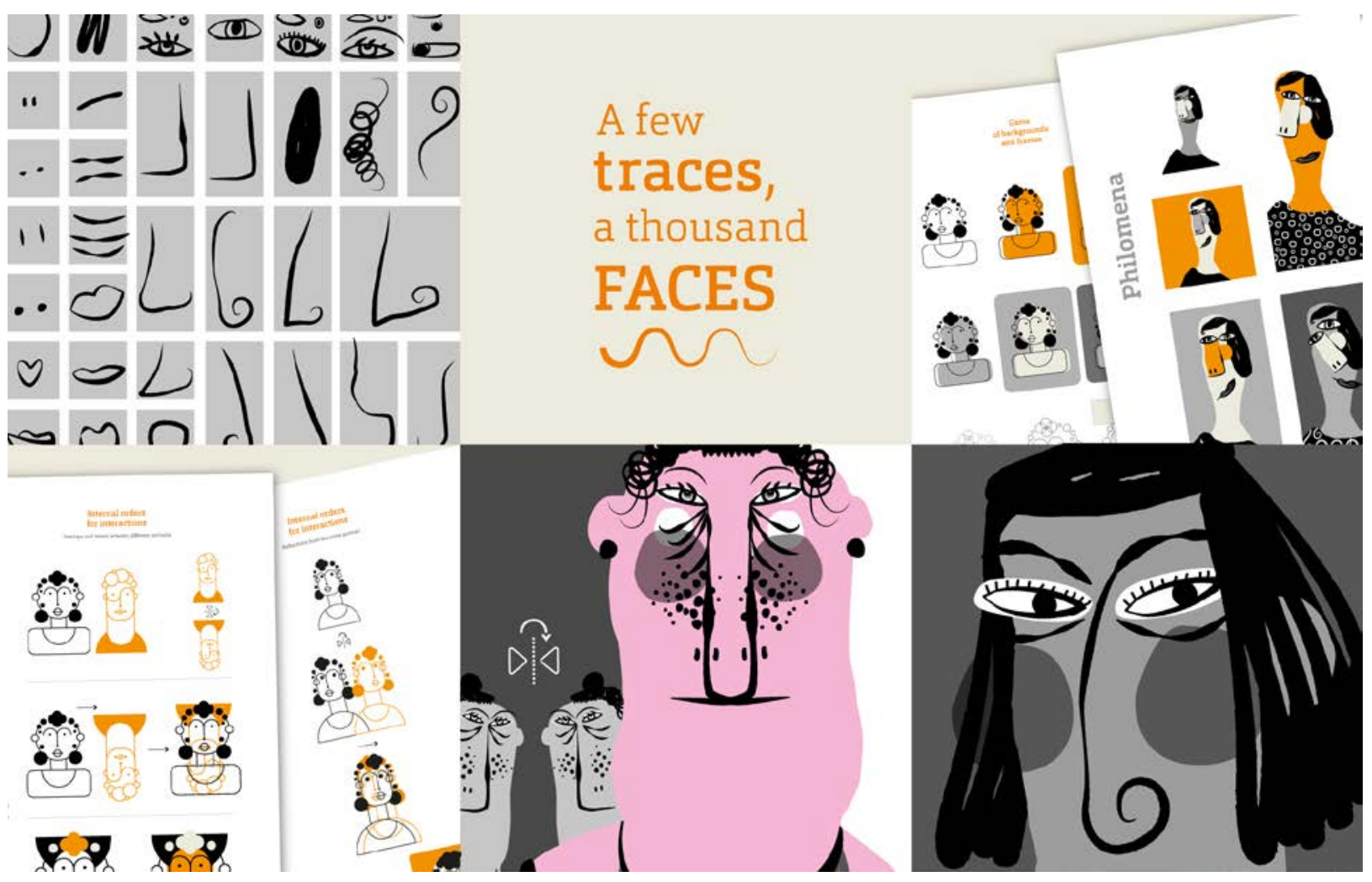

Figura 10 (arriba) y Figura 11 (abajo). El proyecto A few traces, a thousand faces explora las posibilidades del trazo a través del juego y las artes combinatorias. Un grupo de trazados se combina generando 1.000 retratos a partir de reglas y pautas de interacción. Montse Noguera, 2017-2018. Elaboración propia.

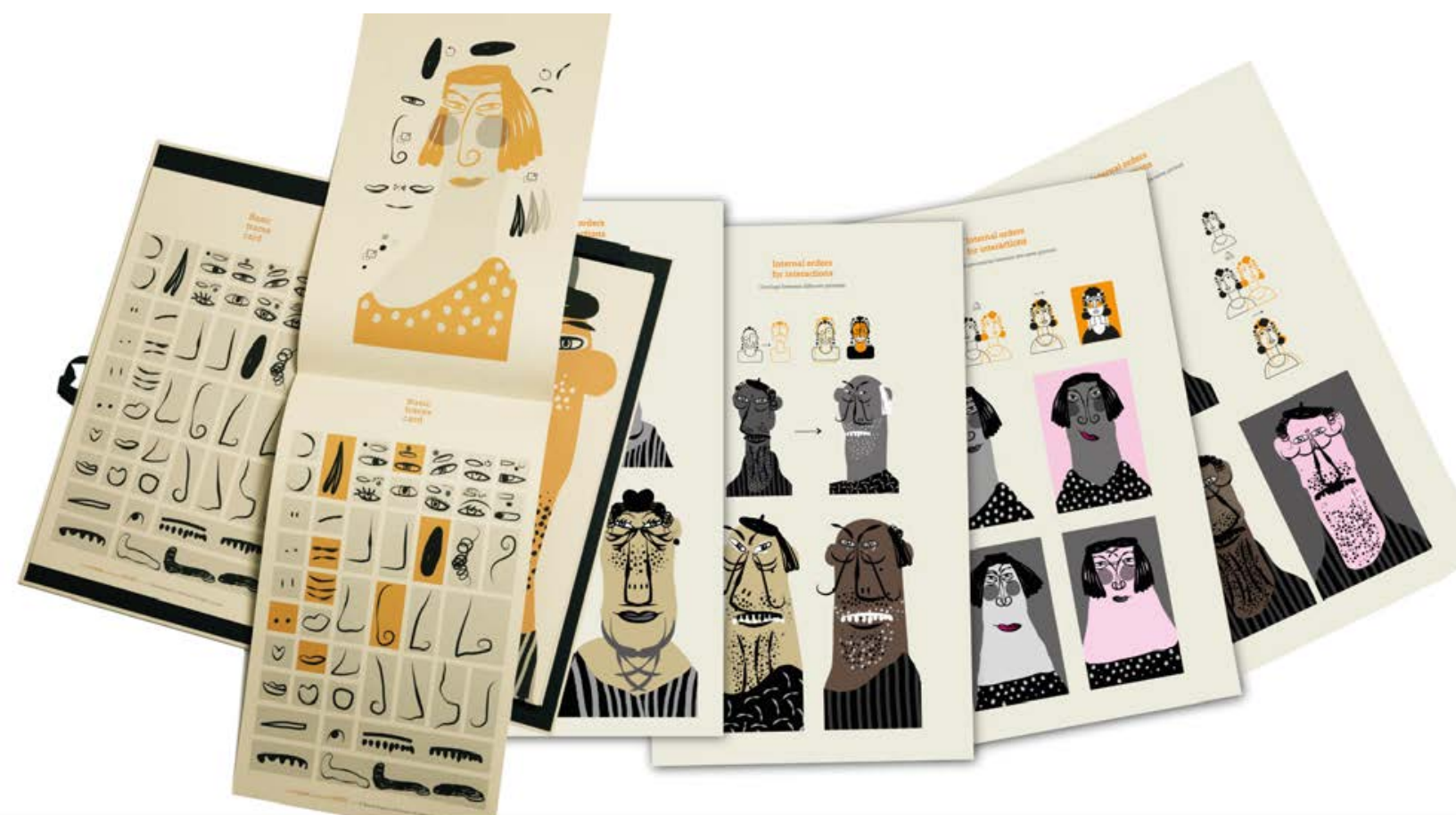

A few traces, a thousand faces. Obra de Montse Noguera Muntadas. 

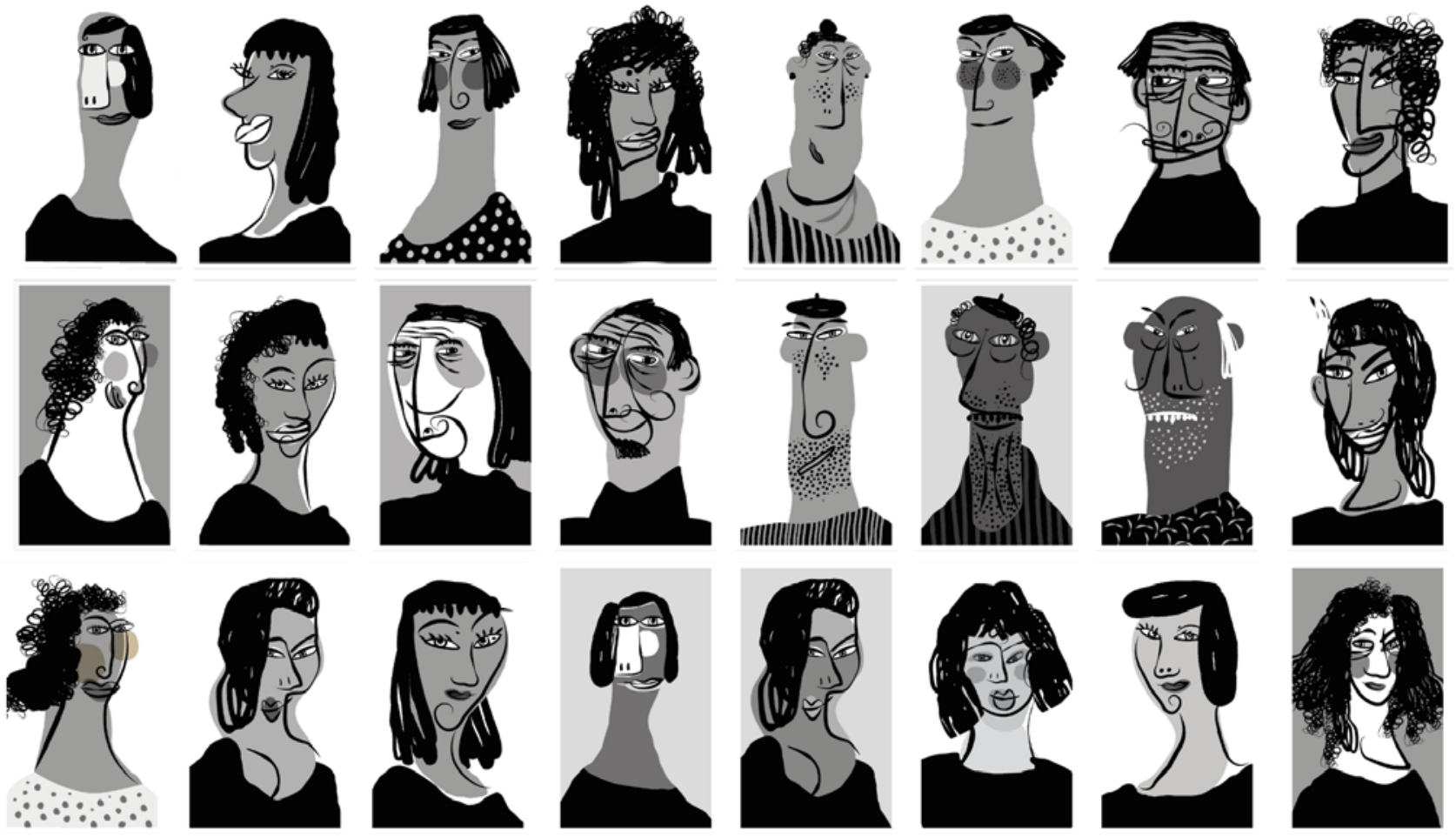

A few traces, a thousand faces. Obra de Montse Noguera Muntadas.

Figura 12. Selección de retratos del proyecto "A few traces, a thousand faces". Montse Noguera, 2018. Elaboración propia.

\section{CONCLUSIONES}

Negroponte apuntaba, en sus estudios sobre lo digital, que: "ser digital es poder crecer" (trad. de Marisa Abdala, 1995: 29). Sin duda podemos usar esa frase a modo de máxima en relación con la metodología más idónea para el aprendizaje formal en el espacio vectorial: el planteamiento del trabajo será más inteligente cuanto más nos proporcione posibilidades de crecimiento, cambio, articulación, y exploración. Huir de la predeterminación es un primer gran paso.

La educación artística busca la profesionalización y a veces resultados a corto plazo, nada de ello es incompatible con lo expuesto. Es posible focalizar la atención en el valor experimental tramando las bases para un modelo de sensibilización de la tecnología y un crecimiento basado en la exploración y la cultura del juego, para que el alumnado pueda continuar su camino sintiendo el gusto por los resultados menos recurrentes o tecnificados. Las escuelas de arte tenemos el poder y el deber, disponemos de la tecnología correcta, podemos acertar en el planteamiento de un aprendizaje que busque continuar con el deseo humano de expresarse con todos los medios a su alcance y sacar el máximo partido de la expresividad y la plasticidad.

"Conocer es el primer paso para amar" (Pla, 1956: 10). Educando para adquirir la libertad que nos da el conocimiento de las cosas, lograremos disfrutar y amar nuestra tecnología.

\section{FUENTES REFERENCIALES}

Codina, L. (2000). El libro digital y la www. Madrid: Tauro producciones.

Huizinga, J. (2007). Homo ludens. Madrid: Alianza Editorial.

Maillard, C. (2009). Contra el arte y otras imposturas. Valencia: Pre-textos. 
Negroponte, N. (1995). El mundo digital. Barcelona: Ediciones B.

Pla, J. (1956). Técnicas de grabado calcográfico. Barcelona: Gustavo Gili.

Ruíz, F. X. (1993). La estética el trazo. El diseño en el diseño. Temes de disseny, 1(9), 117-125.

Tegaldo, O. (2013). El trazo chino y el vacío que lo anima. El Dibujo como soporte aglutinante de la Caligrafía y la Pintura china tradicional. Universidad Politécnica de Valencia. Facultad de Bellas Artes de San Carlos. Departamento de dibujo. 